\title{
Capsule Commentary on Ivlev et al., Use of Patient Decision Aids Increased Younger Women's Reluctance to Begin Screening Mammography: A Systematic Review and Meta-Analysis
}

\author{
Amir Mohammad, MD, MPH, FACPM, FACOEM \\ Yale University School of Medicine and VA Connecticut Healthcare System, New Haven, CT, USA.
}

J Gen Intern Med 32(7):802

DOI: $10.1007 / \mathrm{s} 11606-017-4073-3$

(c) Society of General Internal Medicine 2017

$\mathrm{C}$ ertified decision aids that use evidence-based information are emerging to assist patients making shared medical decisions. In this systematic review and meta-analysis, the authors attempted to address the effectiveness of breast cancer screening patient decision aids (BCS-PtDAs) and their impact on screening mammography rates among younger women. The authors note that breast screening recommendations are not uniform, ${ }^{1-3}$ which may further lead to confusion and inconsistency in following recommended guidelines and potentially to noncompliance among providers.

After going through the rigorous criteria and utilizing the Grading of Recommendations Assessment, Development, and Evaluation (GRADE) approach, the authors included only six studies in their analysis. However, only three studies used controlled randomization, and the other three were beforeafter studies; decision aids evaluated included written material, web-based tools, videos, and other multimedia programs.

Of the six selected studies, three were computerized decision aids and the other three were booklets or pamphlets. This is timely, as several studies have established the effectiveness of PtDAs in shared decision making. ${ }^{4}$ The essential function of communication in cancer screening is to ensure that patients make decisions consistent with their preferences, needs, and values. This study indicates that decision-aid use resulted in significantly fewer young woman (38-50 years old) opting to receive screening mammography compared to those who received usual medical care. This is similar to findings about men's PSA screening preferences before and after viewing a decision aid. ${ }^{5}$
This systematic review adds to our knowledge on the utility of decision aids/tools as described by the authors. However, future studies, including randomized controlled trials (RCT), should be conducted to validate these tools in different patient population and healthcare settings. Furthermore, these types of tools and decision aids should be integrated into the electronic health record (EHR) to improve population health. Clinicians should select and utilize the evidence-based tools that help patients in making screening decisions when no single best choice is available.

Corresponding Author: Amir Mohammad, MD, MPH, FACPM, FACOEM; Yale University School of Medicine and VA Connecticut Healthcare System, New Haven, CT, USA (e-mail: amir. mohammad@yale.edu).

\section{Compliance with Ethical Standards:}

Conflict of Interest: The author has no conflict with any of the material in this manuscript.

\section{REFERENCES}

1. Ivlev I, Hickman EN, McDonagh, MS, and Eden KB. Use of patient decision aids increased younger women's reluctance to begin screening mammography: a systematic review and meta-analysis. J Gen Intern Med. 2017. Doi: 10.1007/11606-017-4027-9.

2. Li J, Shao Z. Mammography screening in less developed countries. Springerplus 2015;4(1):615. doi:10.1186/s40064-015-1394-8.

3. Biesheuvel $\mathbf{C}$, Weige $\mathbf{S}$, Heindel $\mathbf{W}$. Mammography screening: evidence, history and current practice in Germany and other European countries. Breast Care. 2011;6(2):104-109.doi:10.1159/000327493.

4. Sepucha KR, Borkhoff CM, Lally J, Levin CA, et al. Establishing the effectiveness of patient decision aids: key constructs and measurement instrument. BMC Med Inform Decis Mak. 2013; 13 Suppl 2:S12. doi: 10. 1186/1472-6947-13-S2-S12. Review.

5. Barry MJ, Wexler RM, Brackeet CD, et al. Responses to a Decision Aid on Prostate Cancer Screening in Primary Care Practices. Am J Prev Med. 2015; 49(4):520-5.

Published online May 11, 2017 